\title{
The interrater reliability of a routine outcome measure for infants and pre-schoolers aged under 48 months: Health of the Nation Outcome Scales for Infants
}

Peter Brann, Gordana Culjak, Nick Kowalenko, Rosemary Dickson, Tim Coombs, Anne Sved Williams, Elisabeth Hoehn, Simon Davies, Margaret Hoyland and Philip Burgess

\section{Background}

A review of Australian mental health services identified a gap in routine outcome measures addressing social, emotional and behavioural domains for pre-schoolers and infants. The Child and Adolescent Mental Health Information Development Expert Advisory Panel Working Group developed the Health of the Nation Outcome Scales for Infants (HoNOSI), a clinician-reported routine outcome measure for use with those aged under 4 years. Prior psychometric testing showed that the HoNOSI was considered to show face validity, and that it met the standards for concurrent validity and internal consistency.

\section{Aims}

We aimed to investigate the interrater reliability of the HoNOSI.

\section{Method}

Forty-five infant mental health clinicians completed HoNOSI ratings on a set of five case vignettes.

\section{Results}

Quadratic weighted kappa interrater reliability estimates showed the HoNOSI to have Almost Perfect interrater reliability for the HoNOSI total score. Of the 15 scales, one had Moderate, seven had Substantial and seven had Almost Perfect interrater reliability. Ten of the fifteen scales and the total score exceeded the Consensus-based Standards for the Selection of Health Measurement INstruments criteria for interrater reliability $\left(\kappa_{W} \geq 0.7\right)$

\section{Conclusions}

There has been a clear need for a routine outcome measure for use with infants and pre-schoolers. This study provides evidence of interrater reliability. The current findings, combined with the face and concurrent validity studies, support further examination of HoNOSI in real-world settings.

\section{Keywords}

HoNOSI; mental health; outcome measure; infants; interrater reliability

\section{Copyright and usage}

(c) The Author(s), 2021. Published by Cambridge University Press on behalf of the Royal College of Psychiatrists. This is an Open Access article, distributed under the terms of the Creative Commons Attribution licence (http://creativecommons.org/ licenses/by/4.0/), which permits unrestricted re-use, distribution, and reproduction in any medium, provided the original work is properly cited.
In 1990, Jenkins identified an urgent need for a system of indicators to enable clinicians to monitor and evaluate mental healthcare. ${ }^{1}$ One reason identified for not routinely using standard outcome measures was the lack of appropriate instruments. ${ }^{2}$ In 1998, Wing et $\mathrm{al}^{3}$ developed the Health of the Nation Outcome Scales (HoNOS), an instrument covering symptoms, functioning, relationships and environmental issues, ${ }^{4,5}$ which could be used routinely in the UK National Health Service to measure progress toward the target set by the UK Department of Health 'to improve significantly the health and social functioning of mentally ill people. ${ }^{6}$ Since then, the HoNOS and its adaptations for children and adolescents (HoNOSCA) and for those aged $>65$ years (HoNOS65+), have been officially adopted in England, Australia, New Zealand and other European countries. ${ }^{7-10}$

Gowers et $\mathrm{al}^{11}$ developed the HoNOSCA as a set of scales to be used in Child and Adolescent Mental Health Services. ${ }^{12}$ The HoNOSCA has been widely used. ${ }^{4,13-19}$ It was designed to be brief, have a similar structure to the HoNOS and provide a broad, quantitative measure of severity to measure a range of behavioural, symptomatic, social and impairment domains in children and adolescents and it has been found to have sound psychometric properties. ${ }^{11,20,21}$

In Australia, the National Outcomes and Casemix Collection (NOCC) was introduced to provide a suite of measures that support clinical practice and comparisons across services and different consumer populations'. ${ }^{19}$ This saw the implementation of a range of outcome measures to routine clinical practice across age groups. At the core of the NOCC was the family of clinician-rated HoNOS. A review of NOCC ${ }^{22}$ identified a measurement gap for infants and pre-schoolers.

The Australian Child and Adolescent Mental Health Information Development Expert Advisory Panel (CAMHIDEAP) ${ }^{23}$ provides advice to the Australian government on mental health information development in Australian child and adolescent mental health services. Several members of CAMHIDEAP participated in a collaborative international review of the interrater reliability of HoNOSCA. ${ }^{13}$ This study also involved participants from the UK, Denmark, Norway and New Zealand. CAMHIDEAP members, including Dr Peter Brann, Dr Tim Coombs and Dr Sally Merry as the New Zealand representative, commenced conversations with the other participants in the HoNOSCA interrater reliability study about the development of a measure for infants and pre-schoolers, i.e. the Health of the Nation Outcome Scales for Infants (HoNOSI). ${ }^{24}$ Australia and New Zealand, through the CAMHIDEAP, commenced the measure development work, with the initial mapping of domains undertaken by Dr Sally Merry. The CAMHIDEAP had formed a working group to look broadly at routine outcome measures in the very young and supported by the results of the review of the NOCC, took ownership of the HoNOSI initiative, developing and refining a measure suitable for sector consultation and field trialling.

Face validity testing ${ }^{25}$ showed that the HoNOSI was perceived to fill a much needed gap in infant mental health outcome 
measurement for the 0-47 month age group (infants and preschoolers). Following subsequent concurrent validity testing, ${ }^{26}$ the CAMHIDEAP Working Group identified the need to test the interrater reliability of the HoNOSI.

\section{Aims}

This HoNOSI field trial was designed to examine interrater reliability, exploring the degree of agreement among different raters when rating the same case vignettes with the HoNOSI.

\section{Method}

A pilot study with three vignettes was conducted by a panel of CAMHIDEAP Working Group members, along with individuals with expertise in infant mental health or mental health measurement. The pilot study identified that the vignettes were substantially more severe than the real cases in the concurrent validity study. The vignettes' severity was adjusted to accord with the real cases and two more vignettes were added. The current study used the resulting five vignettes. The age and gender of the vignettes were 4 months (female), 9 months (male), 3 years (female), 3 years (female) and 4 years (male). The vignettes can be found within the full HoNOSI Field Trial Report. ${ }^{25}$ Presenting problems included perceived agitation, aggressiveness ( 9 months), a paediatric in-patient referral with feeding problems, sensory underresponsiveness and maternal depression (4 months).

For the current study, participants with expertise in infant and pre-school mental health were asked to rate these vignettes. Ethics and research governance approval was obtained from the relevant ethics committee for participants from the Centre for Perinatal and Infant Mental Health and Child and Youth Mental Health Service in Queensland (HREC Ref: HREC/16/QRCH/424), and for participants from the Eastern Health Child Youth Mental Health Service in Victoria (HREC Ref: LR19/040). A total of 45 people participated in the study; 26 from Queensland and 19 from Victoria. All participants received a brief online training session on the measure, ${ }^{27}$ covering areas such as the rating rules and inclusion and exclusion criteria. Upon providing consent via the online consent form, the five vignettes were independently rated online. Those making the ratings were presented with the vignette and then the HoNOSI glossary, from which the rater selected their preferred rating. Results were analysed with Stata statistical software, version 16.1 for Windows. ${ }^{28}$ Interrater reliability was assessed with quadratic weighted kappa. A copy of the vignettes is available from the corresponding author, upon request.

The HoNOSI ${ }^{24}$ is a 15-item set of scales, rated from 0 to 9 , with 0 indicating no problem, 1 indicating a minor problem requiring no formal action, 2 indicating a mild problem, 3 indicating a problem of moderate severity, 4 indicating a severe to very severe problem and 9 indicating not known or not applicable.

The total score is calculated by summing the first 13 scales, which relate to infant mental health status. Missing data is treated as zero in calculating totals. As with the HoNOS family of measures, clinicians typically classify ratings as 'clinically significant ${ }^{29}$ if a problem area is rated as either a Mild, Moderate or Severe to Very Severe problem (i.e. a rating of 2, 3 or 4). A list of HoNOSI scales can be found in Appendix 1.

The strength of agreement between raters was measured against the COnsensus-based Standards for the Selection of Health Measurement INstruments (COSMIN). ${ }^{30}$ The COSMIN initiative ${ }^{30}$ was developed to provide guidance on the selection of outcome measures for clinical and research applications. ${ }^{31}$ The COSMIN includes a methodology for assessing the interrater reliability of measures, ${ }^{30,32}$ a taxonomy and definitions of measurement properties, ${ }^{33}$ checklists for assessing the methodological quality ${ }^{34}$ and criteria for good measurement properties. ${ }^{31}$ For measures constructed with ordinal ratings (i.e. the rating categories indicate the relative ordering of 'clinical severity'), the gold standard for reporting is the weighted kappa, ${ }^{32}$ where reliability is sufficient if $\kappa_{w} \geq 0.7$. The standards outlined by Landis and Koch ${ }^{35}$ are commonly used in discussions of reliability and will also be reported to both facilitate comparison and support interpretation.

\section{Results}

Of the 45 raters, 39 completed all five vignettes with no missing values. Five raters had a missing rating for one scale with one vignette and one rater had a missing rating for one scale with two vignettes. No HoNOSI scale ratings were rated as 9 (not known/ not applicable). In accordance with the rating rules used by the HoNOSCA, all vignettes were able to have total scores calculated. ${ }^{20}$ Table 1 shows descriptive statistics and the HoNOSI total score distribution for each of the five case vignettes. The percentiles indicate the total score that occurred at that percentage of the distribution. For example, for Levi, a score of 17 was $>10 \%$ of the total scores. A score of 18 was $>25 \%$ of total scores. The 50 th percentile is the median score. Percentiles aid in describing the distribution and spread of total scores for each vignette.

All of the possible HoNOSI ratings $(0,1,2,3,4)$ were utilised for each of the 15 scales in at least one vignette. This demonstrates that the full range of possible scores were used in these vignettes and would contribute to the interrater reliability estimate.

Interrater reliability was assessed by quadratic weighted kappa $\left(\kappa_{w}\right)$ estimates (Table 2). The COSMIN standard for acceptable interrater reliability $\left(\kappa_{w} \geq 0.7\right)^{31}$ was exceeded by 10 of the 15 scales and by the total score. From Table 2, it can be seen that scales 7 and 15 were within 0.01 of the standard. Scales 11 and 14 were within 0.08 of the standard. The confidence intervals for those four scales are wide and overlap the standard. Scale $2 \mathrm{had}$ a very wide confidence interval and was clearly lower than the standard.

The Landis and Koch Strength of Agreement criteria ${ }^{35}$ is shown in the rightmost column. Scale 2 (activity/attention) is only Moderate in agreement $(0.41-0.60)$. However, seven of the scales and the total score have Almost Perfect agreement (0.81-1.00), whereas the remaining seven have Substantial agreement (0.61-0.80).

\section{Discussion}

This study was designed to test the interrater reliability of the HoNOSI. Results show that the level of interrater reliability for the total score was Almost Perfect when measured against the benchmarks outlined by Landis and $\mathrm{Koch}^{35}$ and that it also well exceeds the COSMIN standard for interrater reliability. ${ }^{32}$ The majority of individual scales clearly met the COSMIN criteria and applying the commonly used Landis and $\mathrm{Koch}^{35}$ descriptors, seven scales and the total score had Almost Perfect agreement and seven scales had Substantial agreement between raters.

COSMIN guidelines describe what constitutes a sufficient level of agreement. Using the COSMIN criteria for good measurement properties, the results of the present study well exceed the criteria for interrater reliability, where reliability is rated as either sufficient $\left(\kappa_{w} \geq 0.7\right)$, insufficient $\left(\kappa_{w}<0.7\right)$ or indeterminate (where $\kappa_{w}$ is not reported. Scales 7 (problems associated with regulation and integration of sensory processing), 11 (problems with age-appropriate selfcare and environmental exploration), 14 (problems with knowledge 
Table 1 Distribution of Health of the Nation Outcome Scales for Infants total score for the five vignettes

\begin{tabular}{|c|c|c|c|c|c|c|c|c|c|c|}
\hline \multirow[b]{2}{*}{ Vignette } & \multirow[b]{2}{*}{ Raters, $n$} & \multirow[b]{2}{*}{ Mean } & \multirow[b]{2}{*}{ s.d. } & \multirow[b]{2}{*}{ Minimum } & \multirow[b]{2}{*}{ Maximum } & \multicolumn{5}{|c|}{ Percentiles } \\
\hline & & & & & & 10th & 25th & 50th & 75th & 90th \\
\hline 1 - Levi (9 months) & 45 & 20.7 & 3.9 & 14 & 31 & 17 & 18 & 20 & 22 & 27 \\
\hline 2 - Lily (4 months) & 45 & 17.8 & 4.4 & 12 & 31 & 13 & 15 & 17 & 20 & 23 \\
\hline 3 - Chloe (3 years) & 45 & 10.3 & 3.6 & 2 & 22 & 6 & 8 & 10 & 12 & 13 \\
\hline 4 - Sandy (4 years) & 45 & 31.1 & 4.0 & 26 & 42 & 26 & 28 & 31 & 33 & 35 \\
\hline 5 - Sally (3 years) & 45 & 37.6 & 4.3 & 27 & 46 & 31 & 36 & 37 & 40 & 43 \\
\hline
\end{tabular}

or understanding about the nature of the infant's difficulties) and 15 (problems with lack of information, understanding about services or managing the infant's difficulties) were classed as insufficient in terms of reliability (Table 2), although they are close to the arbitrary cut-off $\left(\kappa_{w} \geq 0.7\right)$.

It is only scale 2 (Problems with activity levels, joint and/or sustained attention), with $\kappa_{w}<0.5$, that was found to be insufficient according to the COSMIN criteria, although assessed as a moderate level of agreement according to the guidelines provided by Landis and Koch. ${ }^{35}$ Aside from scale 2, the other 14 HoNOSI scales and the total score showed a reasonable amount of variability between vignettes. The study had sufficient power to test the null hypothesis at the interrater reliability estimate of 0.5 .

The HoNOSI has links with the HoNOS family of measures, especially the HoNOSCA. ${ }^{11}$ The HoNOSI estimates are stronger than those obtained with those measures. ${ }^{13,15}$ Although the HoNOSCA covers different developmental stages in its use across ages 4-18 years, the HoNOSI, with a much a narrower age range, covers developmental shifts of arguably greater magnitude. Although the interrater reliability was Substantial to Almost Perfect for the majority of the scales and the total score, it would be wise to establish whether this overall acceptable estimate hides less acceptable interrater reliability for different age groups. The lower estimate for attention may reflect a differential functioning of the scale for different age groups. It is possible that the scale can be applied very reliably for 9-month-olds and 3-year-olds, but less so for 18-month-olds.
Vignettes have been found to be a valid tool when measuring the quality of clinical practice. ${ }^{36}$ They remain a key method when wanting to ensure that raters are exposed to exactly the same stimuli. However, vignettes are not the same as clinical practice, and the HoNOSI may perform better or worse with real cases. Although the vignettes covered ages from 4 months to 4 years, there were only five presentations assessed. The middle age group was not covered. Evaluating the HoNOSI in routine clinical practice would supplement the vignette approach by ensuring that a greater range of presentations could be examined. Furthermore, the question of whether the HoNOSI has differing reliability between, for example, infants and pre-schoolers, could be examined with larger sampling.

It is anticipated that future research could explore HoNOSI psychometric properties with respect to other domains and consumer attributes, including the specific nature of presenting problems, diagnostic categories and developmental differences. With regard to the rapid maturation that occurs in this age range, conceptualising and assessing test-retest reliability will be an important task. A face validity study ${ }^{25}$ and concurrent validity field trial ${ }^{26}$ have now been completed. There are other psychometric properties (e.g. sensitivity to change) yet to be investigated. However, the interrater reliability findings are sufficiently encouraging to support the further exploration of the HoNOSI. The HoNOSI is promising, and the very young have been excluded from the routine outcome measurement framework for too long. Whether it proves to be useful in this endeavour requires further exploration of reliability, validity and feasibility and this may be best achieved by examination in routine clinical practice.

\begin{tabular}{|c|c|c|c|c|c|c|}
\hline HoNOSI scale & & $\begin{array}{l}\text { Raters, } \\
n\end{array}$ & $\kappa_{W}$ & $\begin{array}{l}\kappa_{w} 95 \% \mathrm{Cl} \\
\text { lower }\end{array}$ & $\begin{array}{l}\kappa_{W} 95 \% \mathrm{Cl} \\
\text { upper }\end{array}$ & $\begin{array}{l}\text { Strength of } \\
\text { agreement }\end{array}$ \\
\hline 1 & $\begin{array}{l}\text { Problems with disruptive behaviour/irritability/undercontrolled } \\
\text { emotional regulation }\end{array}$ & 45 & 0.86 & 0.77 & 0.94 & Almost perfect \\
\hline 2 & Problems with activity levels, joint and/or sustained attention & 45 & 0.41 & 0.00 & 0.81 & Moderate \\
\hline 3 & Non-accidental self-injury or lack of self-protective behaviours & 44 & 0.85 & 0.79 & 0.92 & Almost perfect \\
\hline 4 & Problems with feeding and eating behaviour & 45 & 0.85 & 0.61 & 1.00 & Almost perfect \\
\hline 5 & Problems with developmental delays & 45 & 0.85 & 0.76 & 0.95 & Almost perfect \\
\hline 6 & Problems with physical illness or disability & 45 & 0.80 & 0.57 & 1.00 & Substantial \\
\hline 7 & $\begin{array}{l}\text { Problems associated with regulation and integration of sensory } \\
\text { processing }\end{array}$ & 43 & 0.69 & 0.35 & 1.00 & Substantial \\
\hline 8 & Problems associated with sleep & 45 & 0.85 & 0.57 & 1.00 & Almost perfect \\
\hline 9 & $\begin{array}{l}\text { Problems with emotional and related symptoms or overcontrolled } \\
\text { emotional regulation }\end{array}$ & 44 & 0.80 & 0.55 & 1.00 & Substantial \\
\hline 10 & Problems with social reciprocity & 45 & 0.87 & 0.76 & 0.98 & Almost perfect \\
\hline 11 & $\begin{array}{l}\text { Problems with age-appropriate self-care and environmental } \\
\text { exploration }\end{array}$ & 45 & 0.62 & 0.27 & 0.98 & Substantial \\
\hline 12 & Problems with family life and relationships & 44 & 0.81 & 0.64 & 0.98 & Almost perfect \\
\hline 13 & Problems with attending care, education and socialisation settings & 44 & 0.71 & 0.27 & 1.00 & Substantial \\
\hline 14 & $\begin{array}{l}\text { Problems with knowledge or understanding about the nature of the } \\
\text { infant's difficulties }\end{array}$ & 45 & 0.66 & 0.34 & 0.97 & Substantial \\
\hline 15 & $\begin{array}{l}\text { Problems with lack of information, understanding about services or } \\
\text { managing the infant's difficulties }\end{array}$ & 44 & 0.69 & 0.49 & 0.88 & Substantial \\
\hline $\begin{array}{l}\text { HoNOSI total } \\
\text { score }\end{array}$ & & 45 & 0.85 & 0.70 & 1.00 & Almost perfect \\
\hline
\end{tabular}


A final caveat: if the HoNOSI continues to demonstrate good enough psychometric properties in future research, it will still share one common feature with other routine outcome measures. Measured change cannot specify the cause of the change. The HoNOSI, HoNOSCA and similar measures identify change but do not independently specify whether this is clearly maturational, treatment-initiated or a result of alteration in the child's environment. Understanding the source of any change, positive or negative, remains the clinical task at an individual level and a research and policy task at the aggregate level. Hopefully, the HoNOSI will facilitate routine dialogue about mental health outcomes for our relatively overlooked infants and pre-schoolers.

Peter Brann, Eastern Health Child and Youth Mental Health Service, Australia; and Monash University, Australia; Gordana Culjak $\mathbb{D}$, Australian Mental Health Outcomes and Classification Network, Health Education and Training Institute, Australia; and Sydney Medical School, University of Sydney, Australia; Nick Kowalenko, Department of Psychological Medicine, Sydney Children's Hospital Randwick, Sydney Children's Hospital Network, Australia; Rosemary Dickson, Child and Adolescent Mental Health Information Development Expert Advisory Panel, Australian Mental Health Outcomes and Classification Network, Health Education and Training Institute, Australia; Tim Coombs, Australian Mental Health Outcomes and Classification Network, Australia; and Illawarra Institute for Mental Health, Australia; Anne Sved Williams, University of Adelaide, Australia; and Women's and Children's Health Network, Australia; Elisabeth Hoehn, Queensland Centre for Perinatal and Infant Mental Health, Child and Youth Mental Health Service, Children's Health Queensland Hospital and Health Service, Australia; Simon Davies, Child and Adolescent Mental Health Service, Western Australia Child and Adolescent Health Service, Australia; Margaret Hoyland, Child and Youth Mental Health Service, Children's Health Queensland Hospital and Health Service, Australia; Philip Burgess Analysis and Reporting, School of Public Health, Faculty of Medicine, The University of Queensland, Australia

Correspondence: Dr Gordana Culjak. Email: gordana.culjak@uni.sydney.edu.au

First received 18 Sep 2020, final revision 25 Feb 2021, accepted 9 Mar 2021

\section{Data availability}

The data that support the findings of this study are available from the corresponding author, G.C. upon reasonable request.

\section{Author contributions}

All authors contributed to the conception and design of the study. P. Burgess conducted the analysis. G.C. prepared the final manuscript. All authors reviewed and approved the final version of the manuscript.

\section{Funding}

The study was undertaken by the Child and Adolescent Mental Health Information Development Expert Advisory Panel (CAMHIDEAP), supported by the Australian Mental Health Outcomes and Classification Network (AMHOCN). CAMHIDEAP and AMHOCN have been funded by the Australian Department of Health through a contract with the Health Education and Training Institute.

\section{Declaration of interest}

None.

Appendix 1: Health of the Nation Outcome Scales for Infants (HONOSI) - list of scales

\begin{tabular}{|c|c|}
\hline Scale 1 & $\begin{array}{l}\text { Problems with disruptive behaviour/irritability/ } \\
\text { undercontrolled emotional regulation }\end{array}$ \\
\hline Scale 2 & $\begin{array}{l}\text { Problems with activity levels, joint and/or sustained } \\
\text { attention }\end{array}$ \\
\hline Scale 3 & $\begin{array}{l}\text { Non-accidental self-injury or lack of self-protective } \\
\text { behaviours }\end{array}$ \\
\hline Scale 4 & Problems with feeding and eating behaviour \\
\hline Scale 5 & Problems with developmental delays \\
\hline Scale 6 & Problems with physical illness or disability \\
\hline Scale 7 & $\begin{array}{l}\text { Problems associated with regulation and integration of } \\
\text { sensory processing }\end{array}$ \\
\hline Scale 8 & Problems associated with sleep \\
\hline Scale 9 & $\begin{array}{l}\text { Problems with emotional and related symptoms or } \\
\text { overcontrolled emotional regulation }\end{array}$ \\
\hline
\end{tabular}

Scale $10 \quad$ Problems with social reciprocity

Scale $11 \quad$ Problems with age-appropriate self-care and environmental exploration

Scale $12 \quad$ Problems with family life and relationships

Scale 13 Problems with attending care, education and socialisation settings

Scale $14 \quad$ Problems with knowledge or understanding about the nature of the infant's difficulties

Scale $15 \quad$ Problems with lack of information, understanding about services or managing the infant's difficulties

\section{References}

1 Jenkins R. Towards a system of outcome indicators for mental health care. Br J Psychiatry 1990; 157: 500-14.

2 Slade M, Thornicroft G, Glover G. The feasibility of routine outcome measures in mental health. Soc Psychiatry Psychiatr Epidemiol 1999; 34(5): 243-9.

3 Wing JK, Beevor AS, Curtis RH, Park SGB, Hadden J, Burns A. Health of the Nation Outcome Scales (HoNOS): research and development. Br J Psychiatry 1998; 172: 11-8.

4 Laugharne R, Eaves S, Mascas A, Psatha K, Dinnis G, Trower J, et al. Desperately seeking outcomes: quantifying the effectiveness of community mental healthcare using Health of the Nation Outcome Scales. BJPsych Open 2018; 4(3): 91-4.

5 Kisely S, Campbell LA, Cartwright J, Cox M, Campbell J. Do the Health of the Nation Outcome Scales measure outcome? Can J Psychiatry 2010; 55(7): 431-9.

6 Department of Health. The Health of the Nation - A Strategy For England. HMSO, 1992.

7 Lovaglio PG, Monzani E. Validation aspects of the Health of the Nation Outcome Scales. Int J Ment Health Syst 2011; 5: 20.

8 Adamis D, Papanikolaou V, Michailidis M, Macdonald AJ. Validation of the Greek version of the Health of the Nation Outcome Scales for Elderly People (HoNOS65+, version 3). Aging Ment Health 2013; 17(2): 258-64.

9 Bilenberg N. Health of the Nation Outcome Scales for Children and Adolescents (HoNOSCA)-results of a Danish field trial. Eur Child Adolesc Psychiatry 2003; 12 (6): $298-302$.

10 James M, Painter J, Buckingham B, Stewart MW. A review and update of the Health of the Nation Outcome Scales (HONOS). BJPsych Bull 2018; 42(2): 63-8.

11 Gowers SG, Harrington RC, Whitton A, Lelliott P, Beevor A, Wing J, et al. Brief scale for measuring the outcomes of emotional and behavioural disorders in children. Health of the Nation Outcome Scales for Children and Adolescents (HoNOSCA). Br J Psychiatry 1999; 174: 413-6.

12 Gowers SG, Bailey-Rogers SJ, Shore A, Levione W. The Health of the Nation Outcome Scales for Child and Adolescent Mental Health (HoNOSCA). Child Psychol Psychiatry Rev 2000; 5(2): 50-6.

13 Hanssen-Bauer K, Gowers S, Aalen OO, Bilenberg N, Brann P, Garralda E, et al. Cross-national reliability of clinician-rated outcome measures in child and adolescent mental health services. Adm Policy Ment Health 2007; 34(6): 513-8.

14 Trauer T. Outcome Measurement in Mental Health: Theory and Practice. Cambridge University Press, 2010.

15 Pirkis JE, Burgess PM, Kirk PK, Dodson S, Coombs TJ, Williamson MK. A review of the psychometric properties of the Health of the Nation Outcome Scales (HoNOS) family of measures. Health Qual Life Outcomes 2005; 3: 76.

16 Brann $\mathrm{P}$, Coleman $\mathrm{G}$. On the meaning of change in a clinician's routine measure of outcome: HoNOSCA. Aust N Z J Psychiatry 2010; 44(12): 1097-104.

17 Duffy F, Skeldon J. A CAMHS Intensive Treatment Service: clinical outcomes in the first year. Clin Child Psychol Psychiatry 2014; 19(1): 90-9.

18 Lesinskiene S, Senina J, Ranceva N. Use of the HoNOSCA scale in the teamwork of inpatient child psychiatry unit. J Psychiatr Ment Health Nurs 2007; 14(8): 727-33.

19 Brann $\mathrm{P}$, Alexander M, Coombs T. Routine outcome measurement in youth mental health: a comparison of two clinician rated measures, HoNOSCA and HoNOS. Psychiatry Res 2012; 200(2-3): 884-9.

20 Pirkis J, Burgess $\mathrm{P}$, Kirk $\mathrm{P}$, Dodson $\mathrm{S}$, Coombs $\mathrm{T}$, Australian Mental Health Outcomes and Classification Network. Review of Standardised Measures Used in the National Outcomes and Casemix Collection (NOCC). NSW Institute of Psychiatry, 2005 (https://www.amhocn.org/sites/default/files/publication_files/review_of_nocc_measures_version_1.2.pdf).

21 Brann P, Coleman G, Luk E. Routine outcome measurement in a child and adolescent mental health service: an evaluation of HoNOSCA. The Health of the Nation Outcome Scales for Children and Adolescents. Aust N Z J Psychiatry 2001; 35(3): 370-6. 
22 National Mental Health Information Development Expert Advisory Pane Mental Health National Outcomes and Casemix Collection: NOCC Strategic Directions 2014-2024. Commonwealth of Australia, 2013 (https://www. amhocn.org/sites/default/files/publication_files/nocc_strategic_directions_20142024.pdf)

23 Australian Mental Health Outcomes and Classification Network. Child and Adolescent Mental Health Information Development Expert Advisory Panel CAMHIDEAP. Australian Mental Health Outcomes and Classification Network 2020 (https://www.amhocn.org/expert-advisory-panels/child-and-adolescentmental-health-information-development-expert-advisory).

24 Commonwealth of Australia for and on behalf of the Australian Mental Health Outcomes and Classification Network. HoNOSI Health of the Nation Outcome Scales for Infants (0-47 months). Glossary. Ver 0.93. Developed under the guidance of the Child and Adolescent Mental Health Information Development Expert Advisory Panel, 2016.

25 Australian Mental Health Outcomes and Classification Network. Health of the Nation Outcome Scales for Infants (HoNOSI) Field Trial Final Report 2020. Australian Mental Health Outcomes and Classification Network, 2020 (https://www.amhocn.org/health-nation-outcome-scales-infants-honosi).

26 Brann P, Culjak G, Kowalenko N, Dickson R, Coombs T, Burgess P, et al. Health of the Nation Outcome Scales for Infants field trial: concurrent validity. BJPSych Open 2021 (in press).

27 Brann P, Coombs T. HoNOSI Training Video. YouTube, 2020 (https://www.you tube.com/watch?v=G9CKBJ-9Ty8\&feature=youtu.be).

28 StataCorp. Stata Version 16.1. StataCorp, 2020 (https://www.stata.com/pro ducts/)

29 Burgess P, Trauer T, Coombs T, Mckay R, Pirkis J. What does 'clinical significance' mean in the context of the Health of the Nation Outcome Scales? Australas Psychiatry 2009; 17(2): 141-8.
30 Consensus-based Standards for the Selection of Health Measurement Instruments (COSMIN). COSMIN Helps You Select the Most Suitable Outcome Measurement Instruments. Amsterdam Public Health, 2018 (https://www.cos $\min . n \mathrm{l} /$ ).

31 Prinsen CAC, Mokkink LB, Bouter LM, Alonso J, Patrick DL, de Vet HCW, et al. COSMIN guideline for systematic reviews of patient-reported outcome measures. Qual Life Res 2018; 27(5): 1147-57.

32 Mokkink LB, Prinsen CAC, Patrick DL, Alonso J, Bouter, L.M., de Vet HCW, et al. COSMIN Methodology for Systematic Reviews of Patient-Reported Outcome Measures (PROMS): User Manual. Version 1.0. Amsterdam Public Health Research Institute, 2018 (https://www.cosmin.nl/wp-content/uploads/ COSMIN-syst-review-for-PROMs-manual version-1 feb-2018.pdf).

33 Mokkink LB, Terwee CB, Patrick DL, Alonso J, Stratford PW, Knol DL, et al. The COSMIN study reached international consensus on taxonomy, terminology, and definitions of measurement properties for health-related patient-reported outcomes. J Clin Epidemiol 2010; 63(7): 737-45.

34 Mokkink LB, de Vet HCW, Prinsen CAC, Patrick DL, Alonso J, Bouter LM, et al. COSMIN risk of bias checklist for systematic reviews of patient-reported outcome measures. Qual Life Res 2018; 27(5): 1171-9.

35 Landis JR, Koch GG. The measurement of observer agreement for categorical data. Biometrics 1977; 33(1): 159-74.

36 Peabody JW, Luck J, Glassman P, Jain S, Hansen J, Spell M, et al. Measuring the quality of physician practice by using clinical vignettes: a prospective validation study. Ann Intern Med 2004; 141(10): 771-80. 